\title{
Regenerative medicine approaches in large animal models for the temporomandibular joint meniscus
}

\author{
Alejandro J. Almarza1', William Chung ${ }^{2}$ \\ 'Departments of Oral Biology and Bioengineering, Center for Craniofacial Regeneration, McGowan Institute of Regenerative \\ Medicine, University of Pittsburgh, Pittsburgh, PA 15213, USA. \\ ${ }^{2}$ Department of Oral and Maxillofacial Surgery, University of Pittsburgh School of Dental Medicine, Pittsburgh, PA 15213, USA.
}

Correspondence to: Prof. William Chung, Oral and Maxillofacial Surgery, University of Pittsburgh School of Dental Medicine, Pittsburgh, PA 15213, USA. McGowan Institute of Regenerative Medicine, University of Pittsburgh, Pittsburgh, PA 15213, USA. E-mail: chungwl@upmc.edu

How to cite this article: Almarza AJ, Chung W. Regenerative medicine approaches in large animal models for the temporomandibular joint meniscus. Stomatological Dis Sci2019;3:6. http://dx.doi.org/10.20517/2573-0002.2019.02

Received: 1 Feb 2019 First Decision: 4 Mar 2019 Revised: 1 Apr 2019 Accepted: 16 Apr 2019 Published: 26 Jun 2019

Science Editor: Jon D. Wagner Copy Editor: Cai-Hong Wang Production Editor: Jing Yu

\begin{abstract}
Several recent advancements haveoccurred regarding engineering replacements for tissues of thetemporomandibular joint (TMJ). This review specifically summarizes the in vitro technological developments for TMJ meniscus tissue engineering followed by an in-depth look at in vivo studies for the regeneration of the TMJ meniscus, with a focus on large animal models. One recent study used mini-pigs to study implants placed into a partial thickness defects of the TMJ meniscus. The authors investigated the use of allogeneic costal chondrocytes in scaffold-free TMJ implants. Implants were found to be well integrated and the mechanical strength of the defects was more robust in the implanted meniscus than in untreated defects. Two recently published studies in a canine model showed that an extracellular matrix bioscaffold derived from porcine urinary bladder was an effective material for reconstruction of the TMJ meniscus. The composition and mechanical properties of the remodeled tissue were similar to that of the native meniscus. It is important to note that the half-life of these replaced meniscus is unknown, and that successful repair is dependent on identifying the cause for internal derangement. In the future, a large animal model that includes both healthy and pathogenic sites of implantation needs further investigation.
\end{abstract}

Keywords: Temporomandibular joint, meniscus, replacement, xenografts, regenerative medicine, perforation, internal derangement

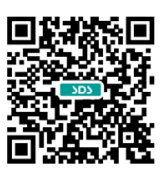




\section{INTRODUCTION}

The most common temporomandibular joint (TMJ) disorder involving the meniscus is internal derangement (ID), where the meniscus is anteriorly displaced and becomes anatomically damaged and/or perforated, potentially requiring a meniscectomy (removal) as a definitive treatment ${ }^{[1]}$. As reviewed by Farrar and $\mathrm{McCarty}^{[2]}$, almost 70\% of patients with temporomandibular dysfunction suffer from meniscus displacement; a finding that identifies the TMJ meniscus as a central factor in the cascade of events that lead to progressive pathology and morbidity. Currently, no alloplastic alternatives exist to safely and effectively replace a degenerative, non-repairable TMJ meniscus. Previous attempts to use alloplastic materials have resulted in unsatisfactory outcomes, including increased joint pathology, among other significant complications ${ }^{[3-6]}$. Several autogenous tissues, such as temporalis muscle, auricular cartilage, dermis, and abdominal adipose tissue, have been used as replacement materials, but only short-term success has been reported ${ }^{[7-13]}$. In addition, the use of these tissues has been associated with donor site morbidity, the eventual formation of scar tissue, decreased range of motion of the mandible, and additional joint pathology. Thus, the identification of an effective off-the-shelf replacement material would represent a significant clinical advance, obviating donor site morbidity and avoiding downstream degenerative changes to the condyle.

\section{IN VITRO TISSUE ENGINEERING OF TMJ MENISCUS REPLACEMENTS}

By 2001, methods for TMJ tissue engineering, which includes cell sources, scaffolding materials, and signaling, were exiguous ${ }^{[14]}$, with the most advances in developing a customized TMJ meniscus scaffold utilizing photopolymerization methods ${ }^{[1,16]}$. From that point there was a shift in TMJ tissue engineering efforts to the use of porcine TMJ meniscal cells for creating a tissue engineered meniscus in vitro. These studies optimized monolayer culture conditions, identified preferred synthetic scaffolds, seeding density and technique, growth factor regimens, and biomechanical stimulation regimens, but the ability to create a meniscus with properties that matched the native structure was limited and evaluation of in vivo remodeling was not conducted. An additional series of studies has been published in which costal cartilage was evaluated as an autologous cell source for a tissue engineered meniscus ${ }^{[17-19]}$.

There have been other efforts on the use of decellularized ECM scaffolds. Lumpkins et al ${ }^{[20]}$ proposed using a decellularized porcine meniscus as a xenogenic scaffold, comparable to approaches seen with other tissues such as heart valves ${ }^{[21]}$. In this study, meniscus decellularized with sodium dodecyl sulfate maintained their size and shape, and displayed similar mechanical energy dissipation characteristics similar to the native meniscus. This study was published very recently, and the future utility of these scaffolds is unknown, as these constructs have yet to be tested with cells.

\section{IN VIVOTISSUE ENGINEERING OF TMJ MENISCUS REPLACEMENTS}

Small animal models (mice) have been used to study meniscus regeneration. However, the small size of the tissues only allows for histological assessment ${ }^{[22-24]}$. The rabbit model offers an intermediate sized tissue for assessment at a moderate price. There has been both full meniscus replacement ${ }^{[25]}$ and partial meniscus replacement ${ }^{[26-29]}$ studies in the rabbit. Again, the size of the animal prevented any mechanical testing of the regenerated tissue, and all results were histological or biochemical.

One recent study used a large animal, where implants were placed into a partial thickness defects of the TMJ meniscus in a mini-pig model ${ }^{[30]}$. Specifically, the authors investigated the use of allogeneic costal chondrocytes for the development of a transplantable, cell-based, scaffold-free TMJ implants. Implants were found to be well integrated and the mechanical strength of the defects was more robust in the implanted meniscus than in untreated defects. These findings were associated with reduced observations of pathologic or abnormal condylar remodeling in treated animals and provided one of the first proof-of-concept studies of a cell-based tissue engineered meniscus replacement in an animal model. 


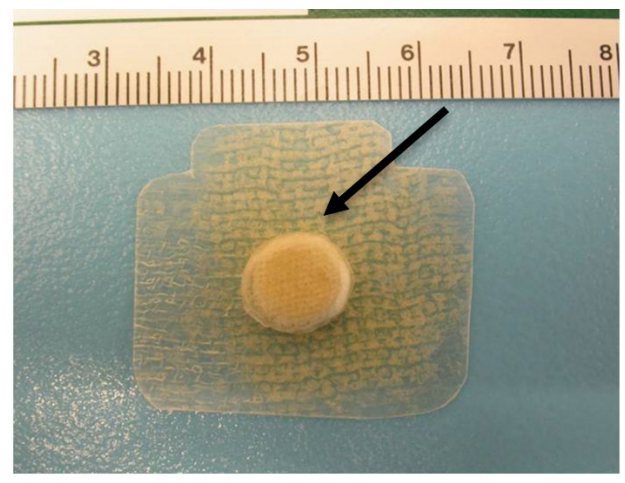

Figure 1. Macroscopic image of the extracellular matrix (ECM) device. The central "pillow" consists of a fine particulate form of the same ECM that encases the deformable pillow. The encasing sheets (arrow) serve as the brim for attachment to the joint capsule. Copyright(c) 2011 (Reprinted with permission)

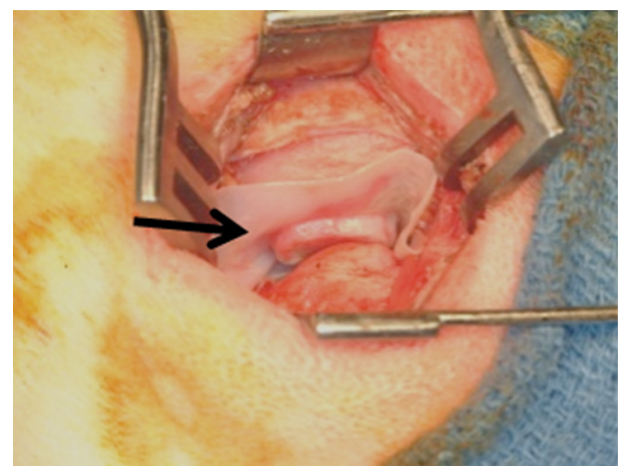

Figure 2. Surgical procedure of extracellular matrix device (arrow) placed between mandibular condyle and temporal fossa. Copyright(c) 2011 (Reprinted with permission)

Two other studies replaced the entire TMJ meniscus in a canine model ${ }^{[31,32]}$. Specifically, the authors showed that an extracellular matrix (ECM) bioscaffold derived from porcine urinary bladder was an effective material for reconstruction of the TMJ meniscus [Figures 1 and 2]. In these studies, a device consisting of a powdered ECM "pillow" encapsulated within sheets of the same material was placed as an interpositional graft after meniscectomy in a canine model. In the first study, the implanted material was observed to progressively remodel from 3 weeks to 6 months after implantation, and the newly formed host tissues resembled the native fibrocartilage of the meniscus in both gross and histologic morphology [Figure 3$]^{[32]}$. In addition to the formation of meniscus-like tissue, the articular surfaces of the temporal bone and mandibular condyle were protected from degenerative changes. A follow-up study of ten dogs demonstrated that the composition and mechanical properties of the remodeled tissue were also similar to that of the native structure ${ }^{[31]}$. Of note, the placement of the device resulted in formation not only of fibrocartilage within the bulk of the implant, but also site-appropriate muscular and ligamentous attachments resembling those found in the native meniscus.

\section{WHY THE CANINE MODEL IS PREFERRED OVER THE PIG AS THE GOLD STANDARD}

As reviewed in a recent publication ${ }^{[33]}$, no one animal model resembles the human TMJ in both structure and function. Nevertheless, the pig has often been described as the "gold-standard" for tissue engineering and regenerative medicine approaches to reconstruction of the TMJ meniscus. However, these assertions are largely based upon post-mortem evaluation. It is important to understand the model-specific advantages and limitations that may exist before embarking into tissue engineering preclinical studies (Figure 4, coronal views of TMJ discs can be found elsewhere ${ }^{[34]}$ ). The advantage of the pig and minipig is that anatomy, 


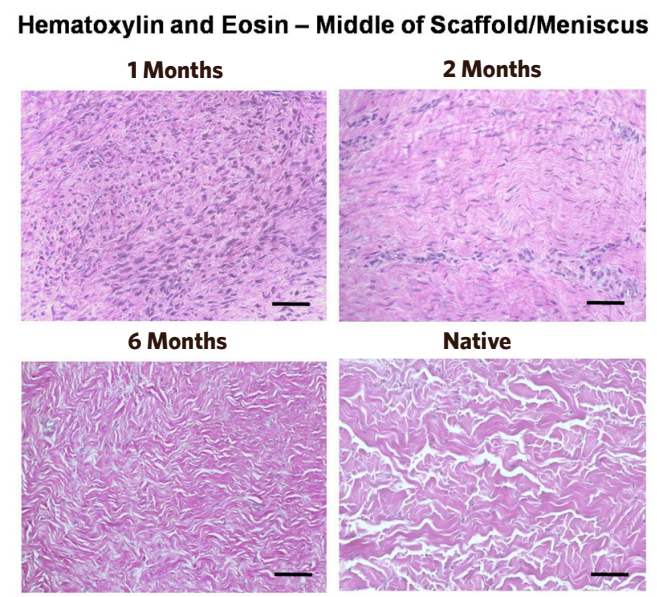

$20 \mathrm{X}$ Magnification, Scale Bar $=100 \mu \mathrm{m}$

Figure 3. Histologic appearance of the remodeling process at 1, 2, and 6 months. The dense cell infiltrate at 1 month transforms into a fibrocartilagenous tissue very similar to the native temporomandibular joint meniscus (lower right). Recall that the original implant is totally acellular ${ }^{[32]}$. Copyright@ 2012 (Reprinted with permission)
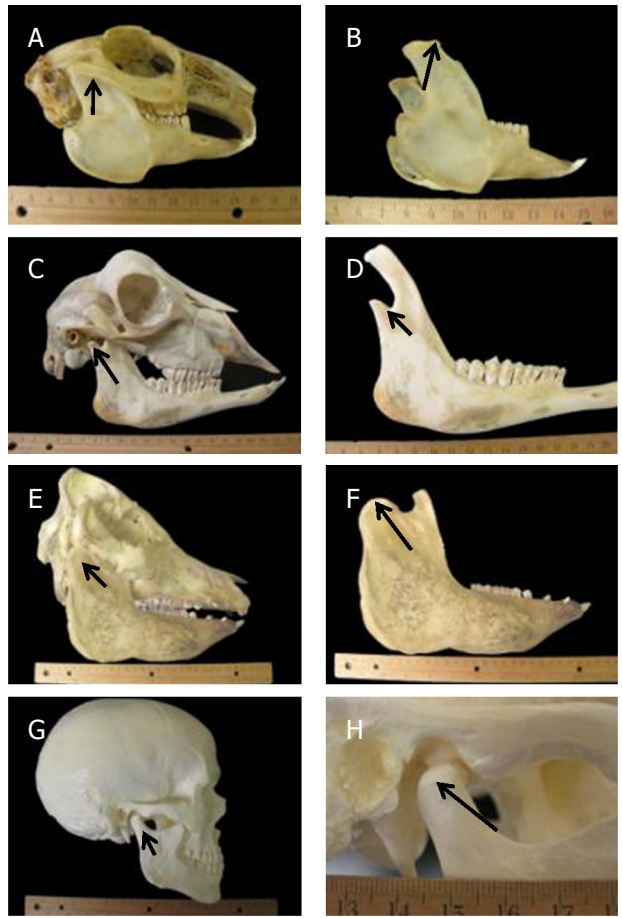

Figure 4. Anatomy of rabbit (A), goat (C), pig (E) and human (G) skulls. Mandibular condyles of the rabbit (B), goat (D), pig (F) and human $(H)$ skulls. Arrows are pointing to the head of the mandibular condyle ${ }^{[44]}$. Copyright@ 2012 (Reprinted with permission)

physiology, and the properties of the tissues have been well characterized. However, the farm pig has the limitation of continuous growth, which confounds results and makes long-term studies not feasible. Also, both in the farm and minipig, the zygomatic arch blocks access into the joint making the surgical approach for TMJ meniscus replacement procedures difficult. The minipig is not widely available, thus obtaining cadaveric tissue and enough animals for a large study may be a challenge. At present, only two farms hold colonies of this breed, and thus access and price can be limitations for investigators. The advantage of the dog is its confined joint space, making attachment of an interpositional device or ECM bioscaffold likely to stay in place. While it is true that the dog is a carnivore, and its TMJ is a hinge joint that can only rotate, the 
type of joint function is not likely to impact the healing potential of the joint. This assertion is supported by recent studies showing similar remodeling in the canine model ${ }^{[31,32]}$. Furthermore, the ECM scaffold technology is a xenogenic implant material, and cross-species implantation is required to evaluate results. Since the majority of FDA approved ECM bioscaffold-based devices are porcine based materials, it is logical to implant porcine based ECM into another species.

Of note, the canine and porcine models of TMJ meniscectomy and replacement are equivalent in their ability to support constructive, site appropriate ECM scaffold remodeling. The pig model requires a young animal for the implantation (less than 3 months and $30 \mathrm{~kg}$ ) as the temporal bone progressively grows ventrally (downward) with age and noticeably limits adequate surgical exposure of the TMJ. In addition, the present studies will include follow up of 12 months post-implantation and the size of the pigs by that age (often greater than $600 \mathrm{lbs}$.) makes husbandry extremely difficult. Finally, the use of pigs would optimally use a xenogeneic source of ECM, likely requiring a canine tissue source, to eliminate the allogeneic variable contribution to remodeling. However, clinical translation will almost assuredly utilize a porcine tissue source of ECM just as most other commercially available ECM scaffold materials utilize, and therefore the dog is a logical xenogeneic model.

\section{MACROPHAGE PHENOTYPE AS A PREDICTOR OF CONSTRUCTIVE REMODELING}

While there are many cell types involved in the inflammatory response, it has been shown that the phenotype of macrophages that participate in the remodeling of ECM scaffolds plays a critical role in determining whether the events follow a pro-inflammatory course leading to fibrosis and scarring, or a constructive and functional remodeling course resulting in deposition and organization of site appropriate tissue ${ }^{[35-40]}$. Although macrophages have been classified as having either an M1 or an M2 phenotype, which is a function of their gene expression pattern, effector molecule production, and biologic function ${ }^{[40,41]}$, the $\mathrm{M} 1 / \mathrm{M} 2$ model is an oversimplification of reality ${ }^{[2]}$. The classically activated "M1" phenotype is the result of in vitro suprastimulation with IFN- $\gamma$ and lipopolysaccharide (LPS, or endotoxin). The "M2" phenotype is a similar laboratory artifact of in vitro IL-4 stimulation. That being said, these spectral extremes of macrophage phenotype provide context for the range of pro-inflammatory effector cells (M1-like) to anti-inflammatory/ regulatory effector cells (M2-like). The phenotype of macrophages stimulated with mammalian ECM that is thoroughly cleansed of cell remnants following decellularization of the source tissue is of an M2-like phenotype, regardless of source tissue ${ }^{[43]}$.

In both canine and porcine models of TMJ reconstruction, the early remodeling of the implanted ECM bioscaffolds is characterized by robust infiltration by mononuclear macrophages. This response has been observed and is characteristic of the remodeling process associated with ECM scaffold materials that have been implanted in all locations investigated to date. Of note, the macrophage response within the TMJ environment is observed to resolve more quickly (i.e., less than one month) than has been observed for other locations (i.e., between two and three months). One significant deficit in our understanding of ECM remodeling is the difference between normal and pathogenic as well as intra-articular versus soft tissue microenvironments as they relate to macrophage polarization and downstream functional outcomes.

\section{CONCLUSION}

In the future, a large animal model that includes both healthy and pathogenic sites of implantation needs further investigation. To-date, few groups have performed in vivo testing of any tissue engineering or regenerative medicine approach to TMJ reconstruction, let alone in a large animal model. While the three studies in large animals have shown viable replacement up to 6 months, there is still a concern that the new tissue could resorb. As such, long term studies that are carried out 9 to 12 months are needed to show that 
the replaced/repaired meniscus is stable. It is also important to note that successful repair is dependent on identifying the cause for ID, otherwise the replacement will likely fail as well.

Attachments of the scaffolds will be an important point of debate in the near future. The attachments will be dependent on the surgical approach, size of the meniscal defect, and the particular material chosen. The ECM scaffolds were designed to be sutured into the zygomatic arch and the surrounding soft tissues. These sutures were fast resorbing, so the new tissue remained in place by growing new attachments to the capsule. Surgeons may choose to anchor the devices to the condyle, since it would be similar to a procedure currently used by some to reposition the meniscus. Nevertheless, any procedure for attachment should be carefully studied in preclinical models.

While this review focused on the use of large animals for the study of meniscus replacement by measuring mechanical properties, there is additional value to smaller animal models for the study of ID. Many more variables can be studied in small species, such as animal age and gender, relevance of nutrition, diet, mastication, and genetics along with the impact on ID. Furthermore, it is difficult to assess function and pain in large animal models. Thus, investigators will have to decide on a balance between the scientific questions being pursued.

\section{DECLARATIONS}

\section{Authors' contributions}

Almarza AJ and Chung W both contributed to the manuscript.

\section{Availability of data and materials}

Not applicable.

\section{Financial support and sponsorship}

None.

\section{Conflicts of interest}

All authors declared that there are no conflicts of interest.

\section{Ethical approval and consent to participate}

Not applicable.

\section{Consent for publication}

Not applicable.

\section{Copyright}

(c) The Author(s) 2019.

\section{REFERENCES}

1. Dolwick MF. Surgical management. Internal Derangements of the Temporomandibular Joint. San Francisco, CA: Radiology Research and Education Foundation; 1983. pp. 167-92.

2. Farrar WB, McCarty WL Jr. The TMJ dilemma. J Ala Dent Assoc 1979;63:19-26.

3. Alonso A, Kaimal S, Look J, Swift J, Fricton J, et al. A quantitative evaluation of inflammatory cells in human temporomandibular joint tissues from patients with and without implants. J Oral Maxillofac Surg 2009;67:788-96.

4. Ferreira JN, Ko CC, Myers S, Swift J, Fricton JR. Evaluation of surgically retrieved temporomandibular joint alloplastic implants: pilot study. J Oral Maxillofac Surg 2008;66:1112-24.

5. Fricton JR, Look JO, Schiffman E, Swift J. Long-term study of temporomandibular joint surgery with alloplastic implants compared 
with nonimplant surgery and nonsurgical rehabilitation for painful temporomandibular joint disc displacement. J Oral Maxillofac Surg 2002;60:1400-11; discussion 11-2.

6. Dolwick MF, Aufdemorte TB. Silicone-induced foreign body reaction and lymphadenopathy after temporomandibular joint arthroplasty. Oral Surg Oral Med Oral Pathol 1985;59:449-52.

7. Dimitroulis G. A critical review of interpositional grafts following temporomandibular joint discectomy with an overview of the dermis-fat graft. Int J Oral Maxillofac Surg 2011;40:561-8.

8. Dimitroulis G. The use of dermis grafts after discectomy for internal derangement of the temporomandibular joint. J Oral Maxillofac Surg 2005;63:173-8.

9. Matukas VJ, Lachner J. The use of autologous auricular cartilage for temporomandibular joint disc replacement: a preliminary report. J Oral Maxillofac Surg 1990;48:348-53.

10. Meyer RA. The autogenous dermal graft in temporomandibular joint disc surgery. J Oral Maxillofac Surg 1988;46:948-54.

11. Pogrel MA, Kaban LB. The role of a temporalis fascia and muscle flap in temporomandibular joint surgery. J Oral Maxillofac Surg 1990;48:14-9.

12. Thyne GM, Yoon JH, Luyk NH, McMillan MD. Temporalis muscle as a disc replacement in the temporomandibular joint of sheep. J Oral Maxillofac Surg 1992;50:979-87; discussion 87-8.

13. Dimitroulis G. Macroscopic and histologic analysis of abdominal dermis-fat grafts retrieved from human temporomandibular joints. J Oral Maxillofac Surg 2011;69: 2329-33.

14. Glowacki J. Engineered cartilage, bone, joints, and menisci. Potential for temporomandibular joint reconstruction. Cells Tissues Organs 2001;169:302-8.

15. Poshusta AK, Anseth KS. Photopolymerized biomaterials for application in the temporomandibular joint. Cells Tissues Organs 2001;169:272-8.

16. Singh M, Berkland C, Detamore MS. Strategies and applications for incorporating physical and chemical signal gradients in tissue engineering. Tissue Eng Part B Rev 2008;14:341-66.

17. Johns DE, Athanasiou KA. Improving culture conditions for temporomandibular joint disc tissue engineering. Cells Tissues Organs 2007;185:246-57.

18. Johns DE, Athanasiou KA. Growth factor effects on costal chondrocytes for tissue engineering fibrocartilage. Cell Tissue Res 2008;333:439-47.

19. Johns DE, Wong ME, Athanasiou KA. Clinically relevant cell sources for TMJ disc engineering. J Dent Res 2008;87:548-52.

20. Lumpkins SB, Pierre N, McFetridge PS. A mechanical evaluation of three decellularization methods in the design of a xenogeneic scaffold for tissue engineering the temporomandibular joint disc. Acta Biomater 2008;4:808-16.

21. Hopkins RA, Jones AL, Wolfinbarger L, Moore MA, Bert AA, et al. Decellularization reduces calcification while improving both durability and 1-year functional results of pulmonary homograft valves in juvenile sheep. J Thorac Cardiovasc Surg 2009;137:907-13, 913e1-4.

22. Embree MC, Chen M, Pylawka S, Kong D, Iwaoka GM, et al. Exploiting endogenous fibrocartilage stem cells to regenerate cartilage and repair joint injury. Nat Commun 2016;7:13073.

23. Puelacher WC, Wisser J, Vacanti CA, Ferraro NF, Jaramillo D, et al. Temporomandibular joint disc replacement made by tissueengineered growth of cartilage. J Oral Maxillofac Surg 1994;52:1172-7; discussion 1177-8.

24. Wu Y, Gong Z, Li J, Meng Q, Fang W, et al. The pilot study of fibrin with temporomandibular joint derived synovial stem cells in repairing TMJ disc perforation. Biomed Res Int 2014;2014:454021.

25. Ahtiainen K, Mauno J, Ella V, Hagstrom J, Lindqvist C, et al. Autologous adipose stem cells and polylactide discs in the replacement of the rabbit temporomandibular joint disc. J R Soc Interface 2013;10:20130287.

26. Tarafder S, Koch A, Jun Y, Chou C, Awadallah MR, et al. Micro-precise spatiotemporal delivery system embedded in 3D printing for complex tissue regeneration. Biofabrication 2016;8:025003.

27. Lai WF, Tsai YH, Su SJ, Su CY, Stockstill JW, et al. Histological analysis of regeneration of temporomandibular joint discs in rabbits by using a reconstituted collagen template. Int J Oral Maxillofac Surg 2005;34:311-20.

28. Chan WP, Lin MF, Fang CL, Lai WF. MRI and histology of collagen template disc implantation and regeneration in rabbit temporomandibular joint: preliminary report. Transplant Proc 2004;36:1610-2.

29. Wang KH, Chan WP, Chiu LH, Tsai YH, Fang CL, et al. Histological and immunohistochemical analyses of repair of the disc in the rabbit temporomandibular joint using a collagen template. Materials (Basel) 2017;10:E924.

30. Vapniarsky N, Huwe LW, Arzi B, Houghton MK, Wong ME, et al. Tissue engineering toward temporomandibular joint disc regeneration. Sci Transl Med 2018;10: eaaq1802.

31. Brown BN, Chung WL, Almarza AJ, Pavlick MD, Reppas SN, et al. Inductive, scaffold-based, regenerative medicine approach to reconstruction of the temporomandibular joint disk. J Oral Maxillofac Surg 2012;70:2656-68.

32. Brown BN, Chung WL, Pavlick M, Reppas S, Ochs MW, et al. Extracellular matrix as an inductive template for temporomandibular joint meniscus reconstruction: a pilot study. J Oral Maxillofac Surg 2011;69:e488-505.

33. Almarza AJ, Brown BN, Arzi B, Angelo DF, Chung W, et al. Preclinical Animal Models for Temporomandibular Joint Tissue Engineering. Tissue Eng Part B Rev 2018; doi: 10.1089/ten.TEB.2017.0341.

34. Kalpakci KN, Willard VP, Wong ME, Athanasiou KA. An interspecies comparison of the temporomandibular joint disc. J Dent Res 2011;90:193-8.

35. Badylak SF, Valentin JE, Ravindra AK, McCabe GP, Stewart-Akers AM. Macrophage phenotype as a determinant of biologic scaffold 
remodeling. Tissue Eng Part A 2008;14:1835-42.

36. Valentin JE, Stewart-Akers AM, Gilbert TW, Badylak SF. Macrophage participation in the degradation and remodeling of extracellular matrix scaffolds. Tissue Eng Part A 2009;15:1687-94.

37. Brown BN, Valentin JE, Stewart-Akers AM, McCabe GP, Badylak SF. Macrophage phenotype and remodeling outcomes in response to biologic scaffolds with and without a cellular component. Biomaterials 2009;30:1482-91.

38. Brown BN, Londono R, Tottey S, Zhang L, Kukla KA, et al. Macrophage phenotype as a predictor of constructive remodeling following the implantation of biologically derived surgical mesh materials. Acta Biomater 2012;8:978-87.

39. Brown BN, Ratner BD, Goodman SB, Amar S, Badylak SF. Macrophage polarization: an opportunity for improved outcomes in biomaterials and regenerative medicine. Biomaterials 2012;33:3792-802.

40. Galdiero M, Mantovani A. Macrophage plasticity and polarization: relevance to biomaterials. Host Response to Biomaterials. London, UK: Academic Press (Elsevier); 2015. pp. 117-30.

41. Mantovani A, Sica A, Sozzani S, Allavena P, Vecchi A, et al. The chemokine system in diverse forms of macrophage activation and polarization. Trends Immunol 2004;25:677-86.

42. Lawrence T, Natoli G. Transcriptional regulation of macrophage polarization: enabling diversity with identity. Nat Rev Immunol 2011;11:750-61.

43. Sicari BM, Dziki JL, Siu BF, Medberry CJ, Dearth CL, et al. The promotion of a constructive macrophage phenotype by solubilized extracellular matrix. Biomaterials 2014;35:8605-12.

44. Hagandora CK, Almarza AJ. TMJ disc removal: comparison between pre-clinical studies and clinical findings. J Dent Res 2012;91:74552. 